\title{
Selective Noise Cancellation using Independent Component Analysis
}

\author{
Jun-Il Sohn \\ Ubiquitous Computing Lab., Samsung Advanced Institute of Technology, P.O.Box 111, Suwon 440-600, Korea
}

\author{
Sang-Woo Ban and Minho Lee \\ School of Electrical Engineering \& Computer Science, Kynngpook National University, \\ 1370 Sankyuk-Dong, Puk-Gu, Taegu 702-701, Korea
}

(Received 9 September 2002; accepted 25 October 2003)

\begin{abstract}
A conventional active noise control (ANC) system adjusts coefficients of an adaptive filter to minimise energy of the input signal at the error microphone. When a desired signal exists with a noise signal around the error microphone, the ANC system removes even the desired signal. In this paper, we propose new ANC systems that selectively cancel only the noise signal in the mixture of signals at a specific location. Blind source separation (BSS), which is based on independent component analysis (ICA) and which separates the desired sound signal from the unwanted noise signal, is used as a preprocessor of the proposed ANC system. In order to enhance the performance of noise separation, we developed a teacher-forced BSS learning algorithm. The teacher signal is obtained from a loudspeaker of the ANC system. Computer simulation results show that the proposed ANC system, in conjunction with the BSS algorithm, effectively cancels only the ship engine noise signal from the linear and convolved signal mixtures, which include the human voice. Additionally, experimental results for practical applications in a real situation show good performance cancelling only the cooling fan noise from the signal mixtures, which include the voice signal.
\end{abstract}

\section{INTRODUCTION}

In general, a noise signal exists simultaneously with desired sounds at the same location. We are able to focus on an interesting sound given a mixture of sounds. This selective attention capability is very useful in engineering contexts and has many potential applications in areas such as communications, array signal processing, and sensor signal processing.

The conventional active noise control (ANC) system removes a noise signal by generating an antinoise signal of equal magnitude and opposite phase. ${ }^{1-3}$ It has been successfully applied to cancel noise signals in aircraft, headsets, and various machines. ${ }^{2}$ However, when a desired signal, such as a speech signal, exists with a noise signal at the same location, the ANC deletes not only the noise signal but also the desired sound signal. Therefore, selective noise cancellation cannot be accomplished by the conventional ANC scheme. If only the noise signal can be cancelled from a mixture of signals, a more efficient active noise control system can be developed. A blind source separation (BSS) algorithm can separate the independent source signals from a mixture without any prior information except the number of independent sources. ${ }^{4-6}$

In order to cancel only the noise signal, the BSS algorithm can be incorporated into the ANC system as a preprocessor to discriminate between the noise signal and the desired sound signal. If the BSS algorithm can separate a noise signal from signal mixtures and the ANC system can successfully generate the antinoise, a more effective ANC system with a selective cancellation function can be developed. This is achieved by removing only the noise signal from the mixture at a particular location. ${ }^{7}$

In this paper, we propose a new active noise control system that removes only the noise signal around a desired loca- tion. Our proposed system employs the BSS algorithm using ICA as a preprocessor to separate an original signal from a mixture of signals. The separated noise signal is fed into the ANC of the proposed system, and the desired signal to be separated by BSS is used as the target signal in the ANC. The coefficients of the ANC are updated to reduce the difference between the target signal and received signal at a specific microphone that is located at the place where silence is required. However, the BSS algorithm will make errors in separating the independent sources. Moreover, the adaptation process of the adaptive filter cannot generate the antinoise signal completely. In this case, cancelling the noise signal through the output of the loudspeaker also generates an additional independent source signal. As a result, the BSS algorithm may fail to separate the signal mixture into the noise signal and desired sound signal through failing to consider the output of the ANC system as the effect of the additional independent source.

In order to enhance the separation performance of the BSS and whole selective attention system, we use an additional microphone in the consideration of the loudspeaker in the ANC system as an additional independent source. Additionally, we use a priori knowledge of the output of the ANC system in constructing the BSS algorithm. The loudspeaker signal output of the ANC system, that is a priori information, is used to find the coefficients of the demixing matrix. This creates a new learning algorithm for the separation of the signal mixtures, or a so-called "teacher-forced BSS learning algorithm."

Computer simulation results show that speech signals successfully remain around a microphone by the selective reduction a ship engine noise. The proposed ANC system, using both an additional microphone and the teacher-forced 